\title{
Users Want Simple Control over Device Selection
}

\author{
Jan M.V. Misker ${ }^{(1)}$ Jasper Lindenberg ${ }^{(2)}$ Mark A. Neerincx ${ }^{(1,2)}$ \\ (1) Delft University of Technology, Faculty of \\ Electrical Engineering and Computer Science, Man \\ Machine Interaction Group. Mekelweg 4, 2628 CD

\section{(2) TNO Human Factors} \\ Kampweg 5, Soesterberg, The Netherlands \\ $\{1$ indenberg, neerincx $\}$ atm. tno.nl
} Delft, The Netherlands. j.m.v.misker@ewi.tudelft.nl

\begin{abstract}
When users want to combine various resources in an ambient intelligent environment in an ad hoc manner, they need to be able to identify and select these resources. We conducted an experiment to study various user interaction styles for combining input and output devices in an ambient intelligent environment. The results show that the test subjects prefer to press a designated button on a device to select it. They least prefer an automatic selection. The main conclusion is that our subjects are willing to spend a bit of time and effort in order to gain control over their environment. This supports the claim that users acting in ambient intelligent environments should always be kept in the loop; systems should take care not to alienate their users when making decisions for them.
\end{abstract}

\section{Introduction}

The user directed, ad hoc combination of resources in an intelligent, connected environment is not widely researched in the ambient intelligence field. Even though user interaction in ambient intelligent environments is an active field of research, such research is often about the user interaction with environments as a whole, often tightly integrated. Such research is of course very valuable, but we believe that the step before, creating such an environment, needs more interest. It requires both user interaction and technical infrastructure/standards/protocols. We hope to draw a bridge from technical properties of ad hoc connection and information exchange to user understandable, dynamic environments.

In this paper we focus on the user interaction needed when combining input and output devices, especially regarding the identification and selection of such devices. The Identification and Selection (IdS) Matrix presented below serves as a framework upon which the design of the user study was based. Five different interaction styles were selected from the IdS matrix, ranging from no user support to full control for the user.

A prominent example of current technology that partly deals with these issues is Bluetooth. As discussed in [5] Bluetooth is one way of connecting devices, in this study we explore alternatives on a more abstract level.

\subsection{Usage scenario: sharing the fun}

The following usage scenario has guided the design of the identification and selection framework. It shows how ubiquitously connected resources make possible new applications, with a focus on applications involving multiple devices and multiple users in a shared environment.

Eric went on a trip to Amsterdam, with George. He took a lot of pictures and shot some video, as did George. A while later Eric meets his friend Fiona at the supermarket. He has his mobile phone with him, on which he has stored a few of the nicest pictures he made in Amsterdam. Fiona likes them a lot, and wants to see more of them on a bigger screen. They agree to go for a coffee in a nearby cafÈ that has a few tables with a display.

In the cafÉ Eric combines the tabletop screen, his mobile phone and his photo database at home in order to view the pictures on the screen, controlling the slideshow with his phone. While showing Fiona the pictures Eric adds some voice comments to them, using his mobile phone.

Later that day George comes to have dinner at Ericís. After dinner they both write annotations to their own pictures. In addition, they create a joint album with a slideshow, for which they combine their collections, Ericís TV screen and two pointing devices.

\subsection{Technical and user interaction issues}

A basic assumption in this research is that the only feasible way to create truly dynamic, interactive environments is to make the resources more or less autonomous. Such an environment is only loosely connected; connections are made on an ad hoc basis when needed. All resources are responsible for their own functional integrity and their own possible roles in the environment.

A major technical problem to be solved is the issue of discovery. Resources need common (ad hoc) network protocols and communication languages for finding each other and exchanging information. For the scope of this paper, this is considered a technically oriented optimization and standardization issue and as such is not the topic of study. A lot of research is devoted to this issue, Schilit and Sengupta [9] provide an up to date overview.

For a user in a intelligent environment, the main issue is that $\mathrm{s} /$ he does not want to be bothered with all kinds of technical details, or in other words not get lost in ambient intelligence [7]. However, because the resources exchange information in a virtual information space, there will be two issues to overcome:

- Identification: resources can be anonymous on a user level, i.e. the user does not have a way to link the information produced/consumed by a resource to the actual, physical device. Identification thus enables users to create a mapping between the virtual information space, in which the resources communicate, and the physical/real world, in which the user communicates. Symmetrically, resources might need to be aware of which user is using it.

- Selection: in many cases there are multiple resources available that are of potential use to the user. How to choose the right one? What is the ërightí one?

In this paper we define identification of a resource as taking place before or during the selection of that resource. 
Table 1: The matrix describing identification and selection combinations.

\begin{tabular}{|c|c|c|}
\hline $\begin{array}{ll}\text { selection } \\
\text { identification }\end{array}$ & $\begin{array}{c}\mathrm{A} \\
\text { automatic }\end{array}$ & $\begin{array}{c}\mathrm{B} \\
\text { manual }\end{array}$ \\
\hline 1 ñ none & & no_ma \\
\hline 2 ñ label & la_au & la_ma \\
\hline 3 ñ signal to device/user & si_au & si_ma \\
\hline
\end{tabular}

This is a fairly strict approach, because a user can identify a resource after it is selected, e.g. a display that turns on and shows a familiar picture. However such late identification only works for certain resources (e.g. output devices), and is not very usable in many cases.

There are many methods conceivable for combining the various resources in an environment, each with certain usability or technical problems or advantages. Sometimes these methods can be similar from a technological perspective but hugely differ regarding the interaction style they induce, or vice versa. A deeper understanding of the relationship between user interaction, usability and technologies involved can guide expectations about user interaction in dynamic environments and will help in choosing where to focus further technological research. The Identification and Selection (IdS) matrix described in [5] can be used to get a firmer grip on these issues.

Identification and selection go hand in hand, selection is only useful when the selected resources are identified. There are many ways to combine identification and selection strategies. In some combinations the selection and identification occur simultaneously. Each specific combination of strategies has specific pros and cons, some are very well suited to each other, some are downright incompatible.

The matrix in resource.

Table $l$ shows the combinations of a few identification and selection strategies (IdS-comboís) that were used in this experiment. It is a subset of the full matrix as described in [5]. The cells are described in detail in section 2.3 below.

\subsection{Related work}

Only a relatively small body of research is devoted to comparing different user interaction methods for dynamically composing ambient intelligent environments. Often the user interaction is a consequence of the technical workings of the system. Sometimes the starting point of the design is the human, but then often there is only one solution developed, aimed at a very specific usage scenario.

The theoretical aspects of users identifying and selecting resources are not well studied yet. Kray et al. [3] briefly describe some identification issues. Wasinger et al. [12] provide an overview of device assignment. Our strategies differ from theirs because we make a distinction between selection through the userís PDA and selection by usage. Furthermore we study the combination of identification and selection more explicit.

Pasman [8] developed a system for matching services based on a user request in natural language. Lindenberg et al. [4] conducted a user study with this system, comparing it to a more traditional approach for service matching employing a hierarchical list. The major difference with the approach described in this paper is that in their approach the focus is on general services, whereas our approach focuses on physical devices.

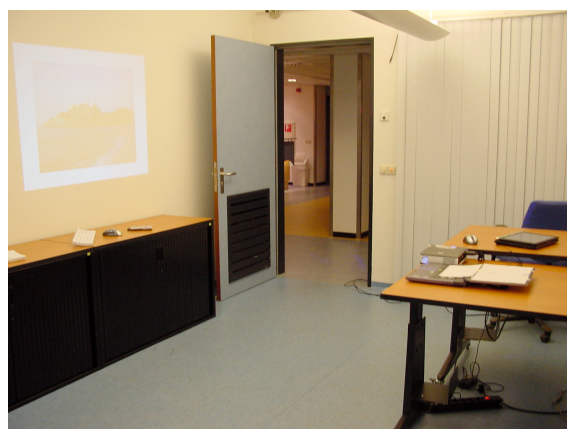

Figure 1: An overview of the experimental setting.

The InfoPoint [2] is a device that can scan visual markers that contain a technical identification code used to address the resource associated with the marker. The InfoPoint further has a get and a put button and its intended use is to support drag and drop operations of data beyond the desktop, amongst devices.

The gesturePen [11] is similar to the InfoPoint, the difference lies mainly in the fact that the gesturePen is used to identify devices, whereas the InfoPoint is used to identify or drop data. The gesturePen was used to a user study, in which it was compared to traditional GUI list selection. The users learned how to use the gesturePen fairly quickly, and indicated they understood the benefit of such identification techniques.

An example of research focusing on the overall user experience is described by Streitz et al. [10]. Many applications and devices are introduced, but a drawback of the approach is that the user interaction studied quickly becomes very specific for a certain application or device. The ad hoc combination as of resources, put forward in this paper, is more general. Furthermore it has the potential to be of better service to the user because they can create new combinations of resources unknown at design time.

\section{Methodology}

\subsection{Goals of the experiment}

The following high level results are aimed for:

- User preference for a certain combination identification and selection strategies.

- User mental model of the environment, is the environment perceived as a tightly orchestrated whole or as independent parts that may or may not cooperate.

- User performance, expressed in timing and number of successful task completions.

- User perception of the dynamics that will occur when multiple users are acting concurrently in the same environment. What will the behavior be like, turntaking? Can social interactions ërepairí the technical problems?

A first consequence of these goals is to do the testing with multiple users at the same time. Furthermore the combinations chosen from the matrix need to be comparable, i.e. they need to be from a small number of rows and columns.

Because the focus of this study is on how users combine resources we did not consider the issue of how the users PDA knows which devices it should look for. For now we assumed and implemented a task oriented description of a group, 
containing a simple list of required roles (services), that the devices can fulfill. Users simply press a button on the PDA to activate such a task.

\subsection{Technical design}

The experiment is set in a homelab setting, see Figure 1. The subjects will use the following equipment:

- 3 displays: 2 tablet PCs, 1 beamer (+ computer).

- 2 Bluetooth keyboards.

- 2 Bluetooth mice.

- 2 Sharp Zaurus PDAís, one for each subject.

All devices have an agent representing them. The user also has a personal agent, running on the PDA, that communicates with the device agents. These agents are implemented in Java, running on the JADE framework. Noteworthy is that the environment is quite varied, Windows, Linux and Mac OS X PCís and laptops are all connected in a WiFi network without too much trouble.

It should be noted that the users will see more agents that offer the required service, this simulates equipment that is not in the nearby vicinity of the user.

The following equipment is used to control the experiment:

- A PC serving as a Bluetooth device host to communicate with the Bluetooth devices.

- Monitoring, logging \& controlling PC.

\subsection{User interaction}

The following IdS-comboís were selected for testing, each causing a different interaction style and as such accompanied by a specific user interface on the PDA (resource.

\section{Table 1):}

- no ma: No identification, manual selection. User is presented with a list of candidates. However no identification is possible, the user thus needs to find out by trial and error which devices are selected.

- la_ma: Label identification, manual selection. User is presented with a list of labeled candidates, separated by role (display, textinput, pointer). User can select the agents that will satisfy the task. Figure 3 depicts the user interface presented on the PDA, the devices carry corresponding labels

- la_au: Label identification, automatic selection. User is presented with a message stating the (labels of the) agents that were selected automatically.

- si ma: Signal identification, manual selection. User can press buttons on devices in the environment. The corresponding agent will be highlighted in the list of candidates on the PDA.

- si_au: Signal identification, (semi-)automatic selection. User can press buttons on devices in the environment. If only one user expressed interest, the selection is made automatically. If multiple users are interested a dialog is presented to all the users, on the PDA. The dialog has two options: yes and no.

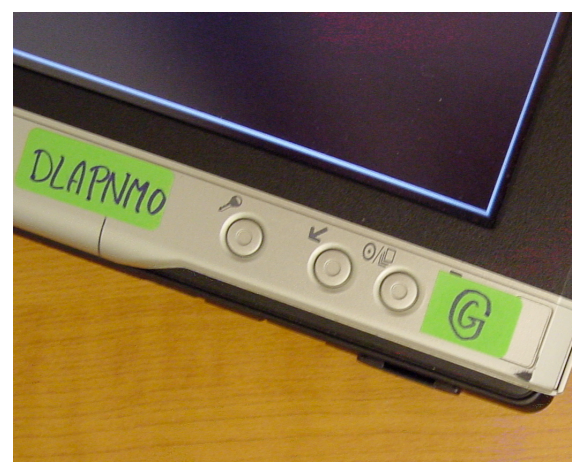

Figure 2: A label and a ëGroupi button on a tablet PC, functioning as a display.

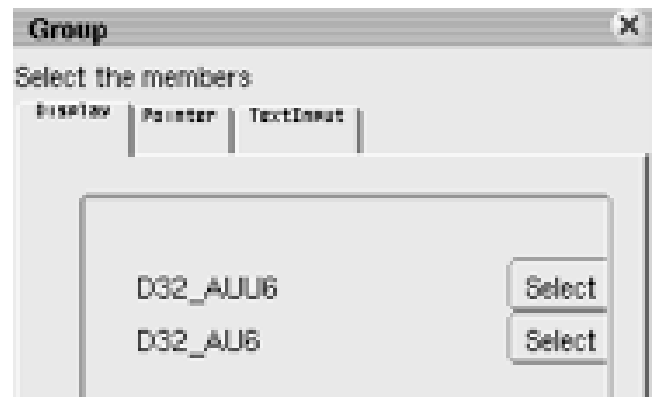

Figure 3: The user interface for label identification and manual selection

Figure 2 shows an example of the labels and buttons used.

Note that besides the user interaction style imposed, these combinations also impose very specific technical requirements. For example labeling usually requires elaborate administration whereas signaling requires all devices to have a ëgroup buttoní. Some aspects of these technical requirements were simulated or mocked-up, but most were implemented as realistic as possible in order to provide the most realistic user experience.

Table 2: Schedule of the experiment.

\begin{tabular}{|c|c|}
\hline User A & User B \\
\hline \multicolumn{2}{|c|}{$\begin{array}{l}\text { general introduction } \\
\text { initial questionnaires }\end{array}$} \\
\hline \multicolumn{2}{|l|}{ explore no_ma } \\
\hline instructions no_ma & explore no_ma \\
\hline \multirow[t]{2}{*}{ test no_ma } & instructions no_ma \\
\hline & test no_ma \\
\hline \multicolumn{2}{|c|}{ questionnaire } \\
\hline \multicolumn{2}{|l|}{ explore la_ma } \\
\hline instructions la_ma & explore la_ma \\
\hline test la_ma & instructions la_ma \\
\hline & test la_ma \\
\hline \multicolumn{2}{|c|}{ dual la_ma } \\
\hline \multicolumn{2}{|c|}{ questionnaire } \\
\hline \multicolumn{2}{|c|}{ final ranking } \\
\hline
\end{tabular}

\subsection{Experimental setup}

A total of 23 subjects conducted the experiment, 16 men and 7 women, aged 19 to 37 .

All of the interaction styles described above were tested with a single and with multiple users, except no_ma because that condition is too hard in the multiple user case. Instead the no_ma style is used to introduce the users to the system. Each 
subject thus had 5 sub-sessions alone, and 4 together with another subject. There was no time limit on the sub-sessions. The duration of the experiment for one pair of subjects was 2 hours. Table 2 shows the flow of the experiment in more detail.

The repeated part is where the conditions are tested. The conditions were presented in a balanced order to the different subjects, excluding the no_ma condition as noted above. Each subject thus used every condition, but in a different order, to prevent ordering effects.

\subsubsection{Photography applications}

The focus of the experiment is on the different interaction styles (IdS-comboís) for combining devices. However to objectively measure that a subject has succeeded in combining the devices in the environment, two applications were designed for the subjects to interact with. While they are making selections, these applications are already running, so they can interact partly already.

The first application is a simple photo annotation application, for which the user has to combine a display (screen), a pointer (mouse) and a text input device (keyboard). This application is used in the single user condition.

For the multiple user condition a photo rating application was used. Two users are presented with the same photo, on a shared display, that they both must rate. A display and two pointers, one for each user, are all that is needed.

\subsubsection{Data collected}

Four types of data are collected during the experiment:

- Questionnaires; at the beginning of the experiment a locus of control questionnaire and a factual technical experience questionnaire are taken. Furthermore after each condition the subjects are asked for their opinion regarding the usability, trust and cooperation of the condition. These are subjective measurements.

- Logfiles; all user interaction with the system is logged. This enables extraction of the time it has taken users to accomplish the tasks, as well as other objective measures.

- Final Ranking; at the end of the experiment the subjects are asked to rank the interaction styles and to provide arguments for the ranking.

- Observations/interview; subjects were encouraged to comment on the experiment, and describe their difficulties with the conditions.

\section{Results}

\subsection{User preference: signal button}

The most prominent result of this experiment is that the majority of the test subject rank the si_au condition the highest, for single user interaction, closely followed by si_ma. The subjects were asked to rank the different conditions at the end of the experiment, separated for single and dual user interaction. The histogram in Figure 4 shows the distribution of ranking scores for single user interaction, Figure 5 for multiple user interaction. It is clear that the no_ma condition, indicated with the white bars, is ranked last by almost all users, making it the least preferred condition. More than half of the subjects rank the si_au condition, black bars, at number one, and another quart rank it at number two. Interestingly, the la_au condition is ranked at the next to last place quite often.

A Friedman test shows that the rankings indeed differ $\left(\chi^{2}\right.$ $=49.113, \mathrm{p}=0.000)$. Wilcoxon Signed Ranks test reveals that indeed the no_ma $(Z=-4.108, p=0.000)$, la_ma $(Z=-2.282$, $\mathrm{p}=0.022)$ and la_au $(\mathrm{Z}=-2.474, \mathrm{p}=0.013)$ rankings differ from the si_au ranking. However the si_ma ranking does not significantly differ $(Z=-0.795, p=0.427)$ from the si_au ranking. Both these conditions employ a signal button, therefore we can safely conclude that the subjects prefer selecting devices using a signal button.

In the multiple user ranking, there are no significant differences between the conditions $\left(\chi^{2}=0.982, \mathrm{p}=0.806\right)$.

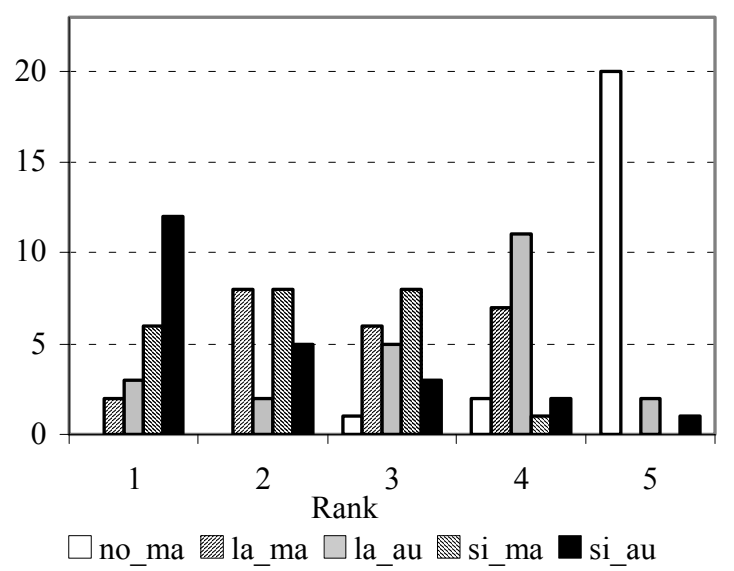

Figure 4: The ranking of the conditions for single user interaction.

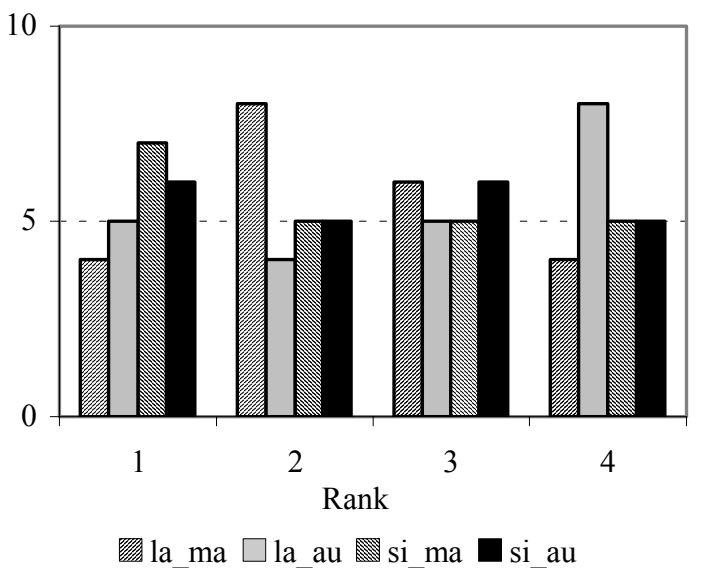

Figure 5: The ranking of the conditions for multiple user interaction. 


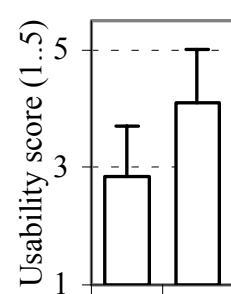

first last

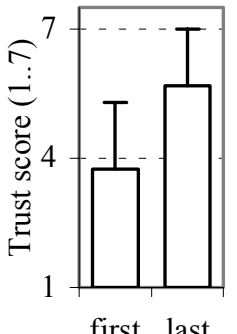

Figure 6: Graphs indicating the development of scores for usability, trust and cooperation.

\subsection{Increasing usability and trust}

The questionnaires are aggregated into one score for usability, trust and cooperation, justified by the high alpha scores for reliability; $0.89,0.90$ and 0.90 respectively.

The subjects gained more trust in the system throughout the experiment, and also their usability scores show an increase. Figure 6 shows the trust and usability score, measured after the first condition and after the last condition. The differences are significant for both usability $(t=5.255, \mathrm{p}$ $=0.000)$ and trust $(\mathrm{t}=5.302, \mathrm{p}=0.000)$. This is interesting, because previous work [6] showed no increase in trust when using mobile devices. The score for cooperation does not change significantly $(t=1.679, p=0.107)$.

The clear ranking preference for si_au, as noted above, is not reflected in the questionnaire scores for usability, trust and cooperation. Figure 8 depicts these scores, per conditon.

A Bonferroni adjusted pairwise comparisons shows that the no ma score for usability is significantly lower than all other conditions except the la_ma condition $(p=0.117)$. All other conditions do not differ significantly from each other at the $\mathrm{p}=0.05$ level, except la_au and si_au $(\mathrm{p}=0.035)$.

The trust scores are very similar, only la_au $(\mathrm{p}=0.001$ and si ma $(p=0.000)$ differ from no ma at the $p=0.05$ level. The cooperation scores do not differ at all at the $p=0.05$ level.

\subsection{User feedback}

The subjects were encouraged to give their feedback on the experimental conditions and on the general concept of ad hoc combining resources. Most of them saw the advantages; some were very enthusiastic and came up with examples of applications themselves, e.g. going to a public library and using the equipment available there combined with a personal device like a mobile phone to work on private projects.

Even though only relatively conventional devices were used in the experiment, the subjects indicated they saw the
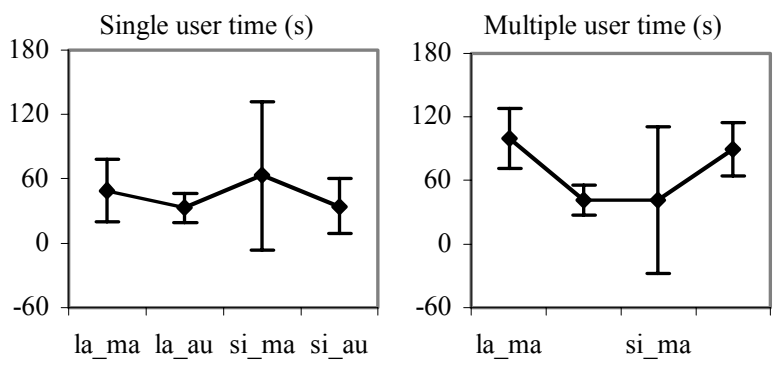

Figure 7: Time till finish of device identification and selection for the single and multiple user cases. The no ma case is left out for reasons of clarity.

advantages of the ad hoc combination of other kinds of devices, like mobile phones, cameraís, printers and so on.

\subsection{No differences in time}

The time it takes subjects to complete the test task has no significant difference between conditions, except for the no_ma condition. A repeated measures analysis with Bonferroni adjusted pairwise comparison reveals only significant differences $(\mathrm{p}<0.05)$ for no ma with la ma $(\mathrm{p}=$ $0.031)$, la_au $(\mathrm{p}=0.007)$ and si_au $(\mathrm{p}=0.014)$, the no_ma and si_ma conditions near $(p=0.057)$ a significant difference. Figure 7 shows that on average subjects took 40 to 50 seconds until they finished setting up the equipment, measured by the time they engaged in the pseudo-task, i.e. annotated the first picture.

The fact that the no_ma condition took all subjects a very long time was expected because (a) the no ma condition provided no support and (b) it was their first exposure to the system.

It should be noted that subjects were not instructed to perform the task as quick as possible, so it is no surprise that the times differ so much.

\subsection{Multiple and single users}

As noted above the rankings do not show a clear winner for the multiple user interaction conditions. Also the cooperation scores do not differ significantly per condition. No effects within the multiple user condition can thus be found in this limited test. Noteworthy is, however, that the ranking for single user interaction differs from the ranking for multiple user interaction, implying that the subjects did have a different preference.

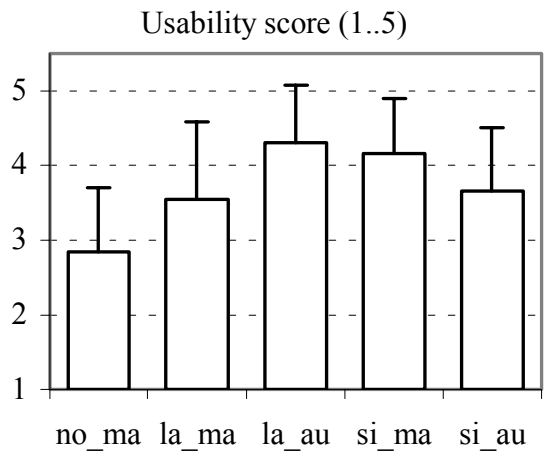

Trust score $(1 . .7)$

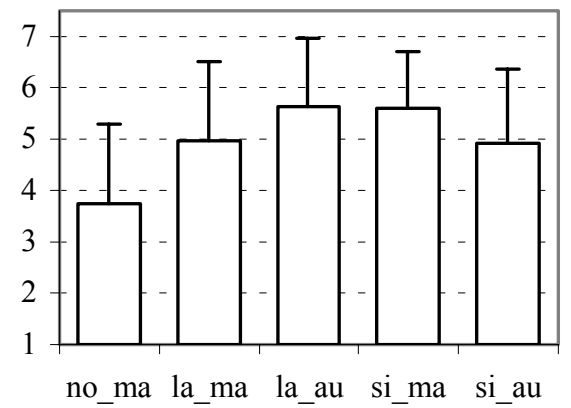

Cooperation score (1..7)

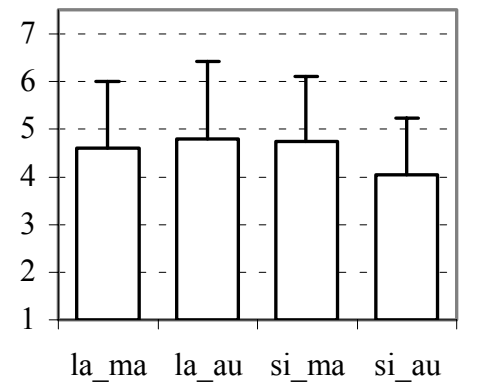

Figure 8: Usability, trust and cooperation scores per condition. 


\subsection{Observation: users expect the same order}

One of the most prominent observations made was that in the manual cases the subjects expected the items on their PDA to be in the same order, for themselves per trial, but most importantly for each other, in the multiple user trials. Which was deliberately not the case, to enforce identification. This is important because even though it is not unfeasible that at least a relative order can be kept constant over time and for all users, e.g. using the devices unique identifier for sorting, but it can never be guaranteed that the same devices will be shown on the lists. Therefore it should be made clear to the users that the list is not ordered, but merely a collection.

\section{Conclusions}

From the rankings it is clear that the majority of the test subjects prefer the si_au condition, closely followed by the si_ma condition. Recall that this condition required them to press a button on a device. From feedback through open questions and their written comments it becomes clear they like the ease of use this provides, keywords were rapid, easy to use and intuitive. The fact that this preference disappears when multiple users are involved can be attributed to the confusion that arises when the conflict resolution popup dialogs are shown on the PDA. We believe that the si au condition can be made more sophisticated to accommodate multiple users better, for example by making the selection process adapt to the situation. The system should perhaps make more clear that there are conflicts, making it worthwhile for the user to do the extra steps.

Another interesting result from the ranking shows that subjects rank the la_au case fairly low. As reasons for this some of them indicated the lack of control as vital for this. Control is in fact the only difference between the si_au and la au condition. Noteworthy is that the only major result from the usability scores is that la_au scores significantly higher than si_au, opposite from the ranking preferences.

Apparently, subjects were quite willing to invest a bit more time and effort in exchange for more control. This result supports our claim that users should always be kept in the loop; systems should take care not to alienate their users when making decisions for them. Even simple tasks, like selecting a mouse, require user interaction.

More research is needed to better take into account multiple user interaction and possibly other interaction styles, e.g. a visual signal on the devices for identification purposes in combination with automatic selection. In our future work we will also focus on the applications that are made possible by ad hoc selection of resources, specifically applications that involve multiple users and more detailed empirically founded scenarios.

\section{Acknowledgements}

The authors thank Stijn Oomes of Delft University of Technology and Jan Willem Streefkerk of TNO human factors for their valuable feedback and help with the statistical analysis.

This research is part of the CACTUS project which is a multi-disciplinary joint research project of Delft University of Technology (Faculty of Industrial Design Engineering and the faculty of Electrical Engineering, Mathematics and Computer Science) and research and technology organization TNO. Parts of the research were supported by the MultimediaN Personal Information Delivery (PID) project.
CACTUS is part of the Freeband Impulse program that aims at the generation of public knowledge in advanced telecommunication (technology and applications). The Dutch Ministry of Economic Affairs is co-funding this program as part of the policy plan "Concurreren met ICT Competenties".

\section{References}

[1] Holmquist, L.E., Mattern, F., Schiele, B., Alahuhta, P., Beigl, M. and Gellersen, H-W. Smart-Its Friends: A Technique for Users to Easily Establish Connections between Smart Artefacts. In Proc. of UbiComp 2001 (Tech note), Atlanta, Georgia, USA, 2001.

[2] Kohtake, N. Rekimoto, J., Anzai, Y. InfoPoint: A Device that Provides a Uniform User Interface to Allow Appliances to Work Together over a Network. Journal of Personal and Ubiquitous Computing, Volume 5 Issue 4, pp. 264-274, December 2001.

[3] Kray, C., Wasinger, R., Kortuem, G. Concepts and issues in interfaces for multiple users and multiple devices, Workshop on Multi-User and Ubiquitous User Interfaces (MU3I) at IUI/CADUI, 2004.

[4] Lindenberg, J., Pasman, W., Kranenborg, K., Stegeman, J., Neerincx, M.A. Improving Service Matching and Selection in Ubiquitous Computing Environments: A User Study. to appear in Personal and Ubiquitous Computing 2005, Springer.

[5] Misker, J.M.V., Veenman, C.J., Rothkrantz, L.J.M. Survey of Device Identification and Selection in Dynamic Environments, technical report MMI2005-1, http://mmi.tudelft.nl.

[6] Neerincx, M.A., Streefkerk, J.W. Interacting in Desktop and Mobile Context: Emotion, Trust and Task Performance. Proceedings of the first European Symposium on Ambient Intelligence (EUSAI), Eindhoven, The Netherlands, 2003.

[7] Nijholt, A., Rist, T., Tuinenbreijer, K. (2004). Lost in ambient intelligence. In: Proceedings ACM Conference on Compuer Human Interaction (CHI 2004), pp. 17251726, ACM, New York.

[8] Pasman, W. (2004). Organizing Ad Hoc Agents for Human-Agent Service Matching. Proc. Mobiquitous 2004 (Boston, MA, August 22-25), 278-287.

[9] Schilit, B.N., Sengupta, U. Device Ensembles, in IEEE Computer, Volume 37 Issue 12, pp 56-64, December 2004.

[10] Streitz, N.A., R`cker, C., Prante, Th., van Alphen, D., Stenzel, R., Magerkurth, C. Designing Smart Artifacts for Smart Environments. IEEE Computer, Vol. 38 No. 3, pp. 41-49, 2005.

[11] Swindells, C., Inkpen, K.M., Dill, J.C., Tory, M. That one there! Pointing to establish device identity. In Symposium on User Interface Software and Technology (UISTí02), pp. 151-160, 2002.

[12] Wasinger, R., Kray, C., Endres, C. Controlling multiple devices, Physical Interaction (PI03) Workshop on Real World User Interfaces at MobileHCI, pp. 60-63, 2003. 